\title{
Synthesis and Spectral Charactrization of Some Pyrimidinones
}

\author{
*Abdulwahab J. Al-Hamdany *Moayed S. Al-Jawady **Rana A. Saeed \\ *Department of Chemistry / College of Science / University of Mosul \\ ** Department of Pharmac/ Technical Institute / Mosul
}

E-mail: ajalhamadany@yahoo.com E-mail: algwady59@yahoo.com

(Received 14 / 5 / 2013 ; Accepted 4 / 8 / 2013)

\begin{abstract}
The phase- transfer catalysis PTC technique was followed to achieve the reaction, using 50\% $\mathrm{NaOH}$ aqueous solution as a base, benzene as a solvent, and tetrabutyl ammonium bromide TBAB as a catalyst, to afford the pyrimidinone compounds.
\end{abstract}

Keywords: Pyrimidinones, Diarylidene acetone.

\section{تشبيد والخواص اللافيه لبصن البيريمينيونات}

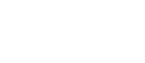

مُ تحضيرسلسلة من الإسيتونات ثنائية الاريلين (42-6) وم تكثيفها مع اليوريا. التبعت ققنية التحفيز بانتق لـ الط -ور لانجاز القاعل بلستخده 50\% محلول هيدروكسيد الصونيوم المائي قاعة والبزين مذيباً وملح بروم الامونيوم رباعي البيوتيل حلوزا لإعطاء مركبت البير لاعيميننيونات.

\section{INTRODUCTION}

Pyrimidinones were synthesised by the reaction of diarylidene acetone (DAA) with urea using $\mathrm{NaOH}$ as a base and bcnzene as a solvent. Diarylidene acetone (DAA) was synthesis by a ClaisenSchmidt reaction. The products always, has at least one double bond conjugated to both a carbonyl group and an aromatic ring (Kappe et al., 1993). Pyrimidinones show versatile biological activities including: anticancer (El-Gazzar et al., 2007), antifungal (Ismail and Koreish, 1992), antimalarial (Tinsley et al., 1981), antibacterial, antihypertensive, antitumor (Kappe et al., 1993), antiinflammatory (Ataby and Eldin, 1999), and antimicrobial effects (Mishra and Moorth, 2008).

\section{EXPERIMENTAL}

Melting points were determined by Gallenkamp apparatus- England Ultra- Violet spectra were obtained using Shimadzu UV- Vissible spectrophotometer UV- 1650.

Fourier-Transform Infrared spectra were recorded on Brucker Tensor spectrophotometer (Japan).

Nuclear magnetic resonance ${ }^{1} \mathrm{H}$ - NMR, ${ }^{13} \mathrm{C}$-NMR spectra were registered at $400 \mathrm{MHz}$ Brucker spectrometer by using tetramethyl silane (TMS) as an internal standard, and $\mathrm{CDCl}_{3}$ as a solvent.

\section{Preparation of Substituted benzalacetone (1-5) (Vogel, 1981): \\ General procedure:}

Place $42 \mathrm{~g}(40 \mathrm{ml})$ of pure substituted benzaldehyde and $63.5 \mathrm{gm}(80 \mathrm{ml})$ of pure acetone in a $250 \mathrm{ml}$, wide- mouthed bottle or bolt- head flask equipped with a mechanical stirrer immerse the reaction vessel in a bath of cold water add slowly from a dropping funnel $10 \mathrm{ml}$ of $10 \%$ Sodium hydroxide solution was added. Render the upper organic layer, extract the lower aqueous layer, wash the later with $20 \mathrm{ml}$ of water and dry with a little anhydrous magnesium sulphate. filtered and 
the solvent evaporated. The physical properties and spectral data of compounds(1-5) were listed in Table (1 and 2).<smiles>CC(=O)[OH2+]</smiles><smiles>CC(=O)/C=C/c1ccc(CC(O)C(C)O)cc1</smiles>

$(1-5)$

Table 1: The physical properties of arylidene acetone (1-5):

\begin{tabular}{|c|c|c|c|c|}
\hline $\begin{array}{c}\text { Compd. } \\
\text { No. }\end{array}$ & $\mathbf{X}$ & $\mathbf{m . p}{ }^{\circ} \mathbf{C}$ & Yield \% & Colour \\
\hline $\mathbf{1}$ & $\mathrm{H}$ & $38-39$ & 71 & Yellow \\
\hline $\mathbf{2}$ & $4-\mathrm{OCH}_{3}$ & $51-53$ & 71 & Yellow \\
\hline $\mathbf{3}$ & $4-\mathrm{NO}_{2}$ & $>300$ & 67 & Violet \\
\hline $\mathbf{4}$ & $4-\mathrm{Cl}$ & $123-124$ & 50 & Pale yellow \\
\hline $\mathbf{5}$ & $4-\mathrm{Br}$ & $193-194$ & 60 & Pale yellow \\
\hline
\end{tabular}

Table 2: The spectral data of arylidene acetone (1-5):

\begin{tabular}{|c|c|c|c|c|c|}
\hline \multirow[b]{2}{*}{$\begin{array}{l}\text { Compd. } \\
\text { No. }\end{array}$} & \multirow{2}{*}{$\begin{array}{l}\mathrm{UV}\left(\mathrm{CHCl}_{3}\right) \\
\lambda \max (\mathrm{nm})\end{array}$} & \multicolumn{4}{|c|}{ I.R(KBr), v cm } \\
\hline & & $C=\mathbf{O}$ str. & $\mathrm{C}=\mathrm{C}$ str. & $\mathrm{C}=\mathrm{C}$ str. & Others \\
\hline 1 & 292 & 1666 & 1626 & 1591 & - . . - \\
\hline 2 & 322 & 1631 & 1610 & 1421 & $\begin{array}{l}\mathrm{C}-\mathrm{O}-\mathrm{C} \\
\text { (sym.,asy)1028,1246 }\end{array}$ \\
\hline 3 & 332 & 1662 & 1595 & 1516 & $\begin{array}{l}\mathrm{N}=\mathrm{O} \text { (sym.,asym.) } \\
1346,1595\end{array}$ \\
\hline 4 & 336 & 1649 & 1601 & 1491 & $\ldots \ldots$ \\
\hline 5 & 340 & 1649 & 1630 & 1487 & - . - - \\
\hline
\end{tabular}

\section{Preparation of diarylidene acetone (DAA)(6-42):}

To a cold stirred mixture of benzaldehyde or substituted benzaldehyde (5.2 gm, 0.03 mole) and benzylideneacetone ( $4.3 \mathrm{gm}, 0.03 \mathrm{~mole})$ in $(50 \mathrm{ml})$ absolute ethanol potassium hydroxide $(1 \mathrm{gm})$ was added in a small portion to the mixture in a period of $(15 \mathrm{~min})$. The stirring was continued for further (1hr) at room temperature (Marrel et al., 1992) and (Vogel, 1981). The physical properties were listed in Table (3). 
<smiles>[Y]c1cccc(/C=C/c2cccc(/C=C/C(=O)/C=C/c3cc(/C=C/C(C)=O)ccc3[X])c2)c1</smiles>

$(6-42)$

Table 3: The physical properties of compounds(6-42)

\begin{tabular}{|c|c|c|c|c|c|}
\hline Compd. No. & $\mathbf{X}$ & $\mathbf{Y}$ & m.p C ${ }^{\circ}$ & Yield \% & colour \\
\hline 6 & $\mathrm{H}$ & $\mathrm{H}$ & $98-100$ & 50 & Yellow \\
\hline 7 & $\mathrm{H}$ & $4-\mathrm{F}$ & $108-110$ & 63 & Yellow \\
\hline 8 & $\mathrm{H}$ & 2,6-diCl & $137-140$ & 34 & Dark Yellow \\
\hline 9 & $\overline{\mathrm{H}}$ & $4-\mathrm{Cl}$ & $108-110$ & 12 & Yellow \\
\hline 10 & $\mathrm{H}$ & $3-\mathrm{NO}_{2}$ & $130-132$ & 90 & Pale Yellow \\
\hline 11 & $\mathrm{H}$ & $3-\mathrm{Br}$ & $89-90$ & 38 & Yellow \\
\hline 12 & $\mathrm{H}$ & $4-\mathrm{Br}$ & $118-120$ & 31 & Yellow \\
\hline 13 & $\mathrm{H}$ & $2-\mathrm{NO}_{2}$ & $90-91$ & 38 & Dark Yellow \\
\hline 14 & $\mathrm{H}$ & $2-\mathrm{Cl}$ & $88-91$ & 40 & Yellow \\
\hline 15 & $\mathrm{H}$ & 2,4-di Cl & $120-122$ & 78 & Pale Yellow \\
\hline 16 & $4-\mathrm{OCH}_{3}$ & $4-\mathrm{F}$ & $90-92$ & 53 & Dark Yellow \\
\hline 17 & $4-\mathrm{OCH}_{3}$ & $3-\mathrm{Br}$ & $118-120$ & 56 & Yellow \\
\hline 18 & $4-\mathrm{OCH}_{3}$ & $2-\mathrm{Cl}$ & $90-93$ & 70 & Yellow \\
\hline 19 & $4-\mathrm{OCH}_{3}$ & $4-\mathrm{Cl}$ & $130-132$ & 94 & Pale Yellow \\
\hline 20 & $4-\mathrm{OCH}_{3}$ & 2,4-diCl & $87-89$ & 50 & Dark \\
\hline 21 & $4-\mathrm{OCH}_{3}$ & $3-\mathrm{NO}_{2}$ & $118-120$ & 20 & Yellow \\
\hline 22 & $4-\mathrm{OCH}_{3}$ & $4-\mathrm{OCH}_{3}$ & $102-104$ & 90 & Pale Yellow \\
\hline 23 & $4-\mathrm{NO}_{2}$ & $2,4-\mathrm{diCl}$ & $120-122$ & 88 & Dark brown \\
\hline 24 & $4-\mathrm{NO}_{2}$ & 4-Cl & $181-184$ & 9 & Dark Yellow \\
\hline 25 & $4-\mathrm{NO}_{2}$ & $4-\mathrm{F}$ & $162-164$ & 13 & brown \\
\hline 26 & $4-\mathrm{NO}_{2}$ & $2-\mathrm{Cl}$ & $247-251$ & 12 & Dark brown \\
\hline 27 & $4-\mathrm{NO}_{2}$ & 2,6-diCl & $115-117$ & 16 & Yellow \\
\hline 28 & $4-\mathrm{NO}_{2}$ & $4-\mathrm{Br}$ & $233-235$ & 8 & Dark brown \\
\hline 29 & $4-\mathrm{NO}_{2}$ & $4-\mathrm{OCH}_{3}$ & $204-206$ & 6 & Dark brown \\
\hline 30 & 4-Cl & 4-Cl & $172-175$ & 72 & Yellow \\
\hline 31 & $4-\mathrm{Cl}$ & $3-\mathrm{Br}$ & $125-126$ & 46 & Pale Yellow \\
\hline 32 & $4-\mathrm{Cl}$ & $2,6-\mathrm{diCl}$ & $157-158$ & 46 & Pale Yellow \\
\hline 33 & $4-\mathrm{Cl}$ & $3-\mathrm{NO}_{2}$ & $159-161$ & 67 & Pale Yellow \\
\hline 34 & $4-\mathrm{Cl}$ & $2,4-\mathrm{diCl}$ & $159-161$ & 77 & Yellow \\
\hline 35 & $4-\mathrm{Cl}$ & $4-\mathrm{F}$ & $152-154$ & 78 & Yellow \\
\hline 36 & $4-\mathrm{Cl}$ & $2-\mathrm{Cl}$ & $123-124$ & 62 & Pale Yellow \\
\hline 37 & 4-Br & $4-\mathrm{OCH}_{3}$ & $178-180$ & 50 & Yellow \\
\hline 38 & $4-\mathrm{Br}$ & $2-\mathrm{Cl}$ & $174-175$ & 25 & Pale Yellow \\
\hline 39 & $4-\mathrm{Br}$ & $4-\mathrm{F}$ & $177-180$ & 50 & Yellow \\
\hline 40 & 4-Br & 4-Cl & $182-184$ & 65 & Yellow \\
\hline 41 & $4-\mathrm{Br}$ & $2,6-\mathrm{diCl}$ & $154-156$ & 91 & Yellow \\
\hline 42 & 4-Br & $3-\mathrm{Cl}$ & $173-174$ & 23 & Pale Yellow \\
\hline
\end{tabular}




\section{Preparation of 4-aryl-6-[(E)-2-arylvinyl]-3,4-dihydropyrimidine-2(1H)-one (General procedure) (Vogel, 1981)}

In a $100 \mathrm{~mL}$ round- bottomed flask equipped with magnetic stirrer, a mixture of (3ml) of $(50 \%)$ sodium hydroxide solution, $(25 \mathrm{ml})$ of benzene, $(0.3 \mathrm{gm})$ of tetrabutyl ammonium bromide (TBAB) and $(0,0025$ mole) of urea was stirred for $(5 \mathrm{~min})$ at room temperature then a $(0.0025$ mole $)$ of (DAA) was added drop wise to the reaction mixture. Stirring was continued at (20-50 $\left.\mathrm{C}^{\circ}\right)$ until no further change in the color of the reaction was observed, the benzene layer was separated and washed three times $(10 \mathrm{~mL})$ of water to remove the base and the catalyst, then was dried over anhydrous magnesium sulfate, filtered and evaporated, finally the residue obtained was recrysallized from ethanol. Some physical properties of these compounds were listed in Table (4).<smiles>[X]c1cccc(/C=C/C(=O)/C=C/c2cccc([X])c2)c1</smiles>

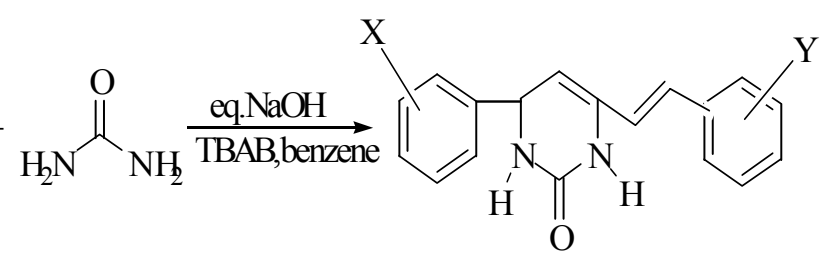

Table 4: The physical properties of compounds (43- 68):

\begin{tabular}{|c|c|c|c|c|c|}
\hline Compd. No. & $\mathbf{X}$ & $\mathbf{Y}$ & m.pc ${ }^{\circ}$ & Yield \% & colour \\
\hline 43 & $\mathrm{H}$ & $3-\mathrm{NO}_{2}$ & $180-183$ & 75 & Dark Yellow \\
\hline 44 & $\mathrm{H}$ & $4-\mathrm{F}$ & $99-100$ & 15 & Yellow \\
\hline 45 & $\mathrm{H}$ & 2,6-diCl & $155-157$ & 20 & Brown \\
\hline 46 & $\mathrm{H}$ & $\mathrm{H}$ & $70-73$ & 50 & Yellow \\
\hline 47 & $\mathrm{H}$ & $4-\mathrm{Cl}$ & $142-144$ & 35 & Orange \\
\hline 48 & $\mathrm{H}$ & $3-\mathrm{Br}$ & $80-82$ & 50 & Yellow \\
\hline 49 & $\mathrm{H}$ & $4-\mathrm{Br}$ & $225-226$ & 33 & Dark Orange \\
\hline 50 & $\mathrm{H}$ & $2-\mathrm{Cl}$ & 159-161 & 50 & Yellow \\
\hline 51 & $\mathrm{H}$ & 2,4-diCl & $131-132$ & 33 & Yellow \\
\hline 52 & $4-\mathrm{OCH}_{3}$ & $3-\mathrm{NO}_{2}$ & $134-136$ & 3 & Brown \\
\hline 53 & $4-\mathrm{OCH}_{3}$ & $4-\mathrm{F}$ & $97-99$ & 25 & Yellow \\
\hline 54 & $4-\mathrm{OCH}_{3}$ & $4-\mathrm{OCH}_{3}$ & $197-198$ & 40 & Yellow \\
\hline 56 & $4-\mathrm{OCH}_{3}$ & $4-\mathrm{Cl}$ & $100-102$ & 57 & Yellow \\
\hline 57 & $4-\mathrm{NO}_{2}$ & 4- $\mathrm{OCH}_{3}$ & $140-142$ & 50 & Yellow \\
\hline 58 & $4-\mathrm{OCH}_{3}$ & 2,4-diCl & $82-83$ & 13 & Dark Yellow \\
\hline 59 & $4-\mathrm{OCH}_{3}$ & $3-\mathrm{Br}$ & $89-90$ & 13 & Yellow \\
\hline 60 & $4-\mathrm{OCH}_{3}$ & $2-\mathrm{Cl}$ & 182-184 & 50 & Yellow \\
\hline 61 & $3-\mathrm{Br}$ & $4-\mathrm{Cl}$ & $140-141$ & 50 & Yellow \\
\hline 62 & 4-Cl & $2-\mathrm{Cl}$ & $78-80$ & 43 & Pale Yellow \\
\hline 63 & 4-Cl & $4-\mathrm{F}$ & $159-161$ & 90 & Yellow \\
\hline 64 & 4-Cl & 2,4-diCl & $143-144$ & 33 & Yellow \\
\hline 65 & $4-\mathrm{Br}$ & $4-\mathrm{OCH}_{3}$ & $156-158$ & 33 & Yellow \\
\hline 66 & $4-\mathrm{Br}$ & $4-\mathrm{F}$ & $65-66$ & 33 & Yellow \\
\hline 67 & $4-\mathrm{Br}$ & $4-\mathrm{Cl}$ & 179-182 & 53 & Yellow \\
\hline 68 & $4-\mathrm{Br}$ & 2,6-diCl & $161-162$ & 33 & Yellow \\
\hline
\end{tabular}


Table 5:The spectral data of compounds (43-68) :

\begin{tabular}{|c|c|c|c|c|c|}
\hline \multirow[t]{2}{*}{ Compd.No. } & \multirow{2}{*}{$\begin{array}{l}\mathrm{UV}\left(\mathrm{CHCl}_{3}\right) \lambda \max \\
(\mathrm{nm})\end{array}$} & \multicolumn{4}{|c|}{ I .R (KBr), v(cm $\left.\mathrm{cm}^{-1}\right)$} \\
\hline & & $\mathrm{C}=\mathrm{O}$ str. & $\mathrm{C}=\mathrm{C}$ str. & N-H str. & Others \\
\hline 43 & 328 & 1645 & 1595 & 3377 & (sym., asy.) $=1344,1408 \mathrm{~N}=\mathrm{O}$ \\
\hline 44 & 340 & 1643 & 1587 & 3242 & \\
\hline 45 & 302 & 1641 & 1639 & 3219 & - . - . - \\
\hline 46 & 336 & 1651 & 1626 & 3417 & - . . - \\
\hline 47 & 262 & 1651 & 1601 & 3346 & $-\ldots$ \\
\hline 48 & 338 & 1645 & 1622 & 3390 & $\ldots$ \\
\hline 49 & 328 & 1616 & 1595 & 3350 &.--- \\
\hline 50 & 332 & 1648 & 1604 & 3296 & $-\ldots$ \\
\hline 51 & 328 & 1620 & 1600 & 3377 & - . - . - \\
\hline 52 & 326 & 1668 & 1622 & 3423 & $\begin{array}{l}\mathrm{N}=\mathrm{O} \text { (sym., asy.) 1350, } 151 \\
\mathrm{C}-\mathrm{O}-\mathrm{C} \text { ( sym., asy.) } 1024,1254\end{array}$ \\
\hline 53 & 350 & 1666 & 1616 & 3480 & $\mathrm{C}-\mathrm{O}-\mathrm{C}$ ( sym., asy.) 1028,1254 \\
\hline 54 & 350 & 1655 & 1631 & 3402 & $\mathrm{C}-\mathrm{O}-\mathrm{C}$ ( sym., asy.) 1030,1252 \\
\hline 55 & 340 & 1650 & 1600 & 3475 & $\mathrm{C}-\mathrm{O}-\mathrm{C}$ ( sym., asy.) 1031,1251 \\
\hline 56 & 352 & 1649 & 1620 & 3419 & $\begin{array}{l}\mathrm{N}-\mathrm{O} \text { (sym., asy.) } 1340,1510 \\
\mathrm{C}-\mathrm{O}-\mathrm{C} \text { ( sym., asy.) } 1254\end{array}$ \\
\hline 57 & 334 & 1668 & 1618 & 3446 & C-O-C (sym., asy.) 1030,1252 \\
\hline 58 & 336 & 1651 & 1604 & 3310 & $\mathrm{C}-\mathrm{O}-\mathrm{C}$ (sym., asy.) 1030,1252 \\
\hline 59 & 350 & 1670 & 1653 & 3064 & $\mathrm{C}-\mathrm{O}-\mathrm{C}$ ( sym., asy.) 1252 \\
\hline 60 & 332 & 1639 & 1617 & 3415 & ---- \\
\hline 61 & 328 & 1640 & 1615 & 3415 & $-\ldots$ \\
\hline 62 & 332 & 1670 & 1626 & 3413 & $-\ldots$ \\
\hline 63 & 328 & 1653 & 1616 & 3435 & ---- \\
\hline 64 & 340 & 1650 & 1598 & 3416 & $\mathrm{C}-\mathrm{O}-\mathrm{C}=$ ( sym., asy.) 1032,1250 \\
\hline 65 & 338 & 1649 & 1624 & 3384 & ---- \\
\hline 66 & 330 & 1653 & 1601 & 3394 & ---- \\
\hline 67 & 332 & 1649 & 1603 & 3417 & ----- \\
\hline 68 & 332 & 1649 & 1581 & 3417 & $-\ldots$ \\
\hline
\end{tabular}


Abdulwahab J. Al-Hamdany et al.

Table 6: ${ }^{1}$ H-NMR spectral data for pared compounds (46-64):

\begin{tabular}{|c|c|c|c|c|c|c|c|c|}
\hline $\begin{array}{l}\text { Comp } \\
\text { d. No. }\end{array}$ & Structures & $\begin{array}{c}\text { Methylene } \\
\text { Protons } \\
\left(\mathrm{C}_{5}\right),(2 \mathrm{H}) \\
\end{array}$ & $\begin{array}{c}\text { Methine } \\
\text { Proton } \\
\left(\mathrm{C}_{6}\right),(1 \mathrm{H}) \\
\end{array}$ & $\begin{array}{c}\text { Nitrogen } \\
\text { Proton } \\
\text { (1H) }\end{array}$ & $\begin{array}{l}\text { Olefinic } \\
\mathrm{C}_{1} `(1 \mathrm{H})\end{array}$ & $\begin{array}{l}\text { Protons } \\
\mathrm{C}_{2^{\prime}}(1 \mathrm{H})\end{array}$ & $\begin{array}{l}\text { Aromatic } \\
\text { Protons }\end{array}$ & $\underset{\text { Protons }}{\mathrm{OCH}_{3}}$ \\
\hline 46 & & $(2.4),(\mathrm{m})$ & (3.3),(s) & (4.1),(s) & $(6.9),(d)$ & $(7.2),(d)$ & $(7-7.6),(m)$ & --- \\
\hline 48 & & $(2.4),(m)$ & (3.4),(s) & (4.4),(s) & $(6.4),(d)$ & $(6.8),(d)$ & $(7.6-7.8),(\mathrm{m})$ & ----- \\
\hline 51 & & $(2.2),(\mathrm{m})$ & (3.4),(s) & (4.5),(s) & (6.1),(d) & (6.8),(d) & $(7.2-7.4),(\mathrm{m})$ & - - - _ \\
\hline 53 & $\widehat{\mathrm{OCH}_{3}}$ & $(2.4),(m)$ & (3.4),(s) & $(4.5),(\mathrm{s})$ & (6.9),(d) & $(7.2),(d)$ & $\begin{array}{c}(7.68-7.70) \\
(\mathrm{m})\end{array}$ & $\begin{array}{l}(3.8) \mathrm{s}, \\
(3 \mathrm{H})\end{array}$ \\
\hline 54 & $\widehat{\mathrm{OCH}_{3}}$ & $(2.3),(\mathrm{m})$ & $(3.6),(\mathrm{s})$ & (3.7),(s) & $(6.2),(d)$ & $(6.9),(d)$ & $\begin{array}{c}\text { (7.68-7.41) } \\
(\mathrm{m})\end{array}$ & $\begin{array}{c}(3.8) \mathrm{s}, \\
(6 \mathrm{H})\end{array}$ \\
\hline 55 & & $(2.1),(\mathrm{m})$ & $(3.5),(\mathrm{s})$ & (3.7),(s) & $(6.9),(d)$ & $(7.1),(d)$ & $(7.5-7.6),(\mathrm{m})$ & $\begin{array}{c}(3.87) \mathrm{s},(3 \\
\mathrm{H})\end{array}$ \\
\hline 57 & $\mathrm{OCH}_{3}$ & $(2.2),(\mathrm{m})$ & (3.7),(s) & (4.4),(s) & $(6.5),(d)$ & $(7.1),(d)$ & $\begin{array}{c}(7.59- \\
7.67),(\mathrm{m})\end{array}$ & $\begin{array}{c}(3.88) \mathrm{s} \\
(3 \mathrm{H})\end{array}$ \\
\hline 60 & $\mathrm{Cl}$ & $(2.4),(\mathrm{m})$ & (3.4),(s) & (4.4),(s) & (6.6),(d) & $(7.1),(d)$ & $(7.8-7.9),(\mathrm{m})$ & $-\ldots$ \\
\hline 61 & & $(2.2),(\mathrm{m})$ & (3.2),(s) & (4.4),(s) & (6.7),(d) & (6.8),(d) & $(7.9-8.3),(\mathrm{m})$ & --- \\
\hline 63 & & $(2.3),(m)$ & (4.8),(s) & (4.8),(s) & (6.8),(d) & (7.1),(d) & $(7.3-7.4),(\mathrm{m})$ & \\
\hline
\end{tabular}


Synthesis and Evaluation

Table 7: ${ }^{13} \mathrm{C}$-NMR data for some of the prepared compounds (Pyrimidinones)

\begin{tabular}{|c|c|c|c|c|c|c|}
\hline $\begin{array}{l}\text { Compd. } \\
\text { No. }\end{array}$ & Structures & \begin{tabular}{|l|l|} 
Olefinic \\
Carbons \\
\end{tabular} & $\begin{array}{l}\text { Aliphatic } \\
\text { Carbons }\end{array}$ & $\begin{array}{c}\text { Aromatic } \\
\text { Carbons }\end{array}$ & $\begin{array}{l}\text { Carbonyl } \\
\text { Carbons }\end{array}$ & $\mathrm{OCH}_{3}$ \\
\hline 48 & & $\begin{array}{l}C_{1}^{\prime}, C_{2}^{\prime}(128,129) \\
C 4(126)\end{array}$ & $\begin{array}{l}\mathrm{C}_{5}(123) \\
\mathrm{C}_{6}(127)\end{array}$ & $(130-143)$ & 188.5 & - \\
\hline 51 & & $\begin{array}{l}C_{1}^{\prime}, C_{2}^{\prime}(130,132) \\
C 4(126)\end{array}$ & $\begin{array}{l}\mathrm{C}_{5}(127) \\
\mathrm{C}_{6}(128)\end{array}$ & 134-145 & 193 & 一 \\
\hline 53 & & $\begin{array}{l}C_{1}^{\prime}, C_{2}^{\prime}(114,131) \\
C 4(161)\end{array}$ & $\begin{array}{l}C_{5}(76) \\
C_{6}(77)\end{array}$ & $(142,143)$ & 188.5 & 55 \\
\hline 55 & & $\begin{array}{l}C_{1}^{\prime}, C_{2}^{\prime}(132,133) \\
C 4(123)\end{array}$ & $\begin{array}{l}\mathrm{C}_{5}(125) \\
\mathrm{C}_{6}(127)\end{array}$ & $(141-143)$ & 188.5 & 55 \\
\hline 57 & & $\begin{array}{l}C_{1}^{\prime}, C_{2}^{\prime}(130,141) \\
C 4(123)\end{array}$ & $\begin{array}{l}\mathrm{C}_{5}(125) \\
\mathrm{C}_{6}(127)\end{array}$ & $(142-143)$ & 161 & 55 \\
\hline 60 & & $\begin{array}{l}C_{1}^{\prime}, C_{2}^{\prime}(129,130) \\
(-) C 4\end{array}$ & $\begin{array}{l}\mathrm{C}_{5}(127) \\
\mathrm{C}_{6}(128)\end{array}$ & $(137-138)$ & 188 & 一 \\
\hline 61 & & $\begin{array}{l}C_{1}^{\prime}, C_{2}^{\prime}(132,133) \\
C 4(127)\end{array}$ & $\begin{array}{l}\mathrm{C}_{5}(127) \\
\mathrm{C}_{6}(128)\end{array}$ & $(134-139)$ & 一 & 一 \\
\hline 63 & & $\begin{array}{l}C_{1}^{\prime}, C_{2}^{\prime}(132,133) \\
C 4(128)\end{array}$ & $\begin{array}{l}\mathrm{C}_{5}(127) \\
\mathrm{C}_{6}(130)\end{array}$ & $(141.5-141.9)$ & 197 & - \\
\hline 64 & & $\begin{array}{l}C_{1}^{\prime}, C_{2}^{\prime}(131,132) \\
C 4(128)\end{array}$ & $\begin{array}{l}\mathrm{C}_{5}(127) \\
\mathrm{C}_{6}(130)\end{array}$ & $(141-143)$ & 188.8 & 55 \\
\hline
\end{tabular}




\section{RESULTS AND DISCUSSION}

The structures of the products were established on the basis of spectroscopic data as in Table (2,4 and 5). The (IR) spectra of compounds(1-6) showed bands in the range of (1670-1616) $\mathrm{cm}^{-}$ ${ }^{1}$ which belongs to the stretching vibration of carbonyl group $(\mathrm{C}=\mathrm{O})(\mathrm{Al}-$ Hajjar and Jarrar, 1980), while the bands in the range (1653-1581) $\mathrm{cm}^{-1}$ related to stretching vibration of carbon- carbon double bond $(\mathrm{vC}=\mathrm{C})$, other broad absorption bands appeared in the range of (3480-3219) $\mathrm{cm}^{-1} \mathrm{due}$ to the stretching vibration of $(\mathrm{N}-\mathrm{H})$ bond, The compound (54) is taken as a representative model for the discussion of the ${ }^{1} \mathrm{H}-\mathrm{NMR}$ spectra.

The ${ }^{1} \mathrm{H}-\mathrm{NMR}$ spectrum of compound (63), showed a multiplet signal at $\delta(2.3) \mathrm{ppm}(2 \mathrm{H})$ attributed to the protons of methylene group at $\mathrm{C}_{5}$ of pyrimidinone ring. Another signal at $\delta(3.6)$ ppm $(1 \mathrm{H})$ referred to the benzylic protone at $\mathrm{C}_{6}$ of pyrimidinone ring and singlet signal at (3.7) ppm related the proton attached to nitrogen while the singlet signal at $\delta(3-8) \mathrm{ppm}(6 \mathrm{H})$ referred to the methoxy group protons, The doublet signal at $\delta(6.8) \mathrm{ppm}(1 \mathrm{H})$ referred to the olefinic proton at $\mathrm{C}_{1}$ of styryl group. Finally, the multiple signal at ${ }^{\delta}(6.9,7.7) \mathrm{ppm}$ referred to the olefinic proton at $\mathrm{C}_{2}$ of styryl group and aromatic proton Table (5). The ${ }^{13} \mathrm{C}-\mathrm{NMR}$ spectrum of compound (55), showed a line at $\delta(141-143) \mathrm{ppm}$ due to the aromatic carbons. Finally, the line at $\delta(188) \mathrm{ppm}$ attributed to the carbonyl carbon. Table (6).

The ${ }^{1} \mathrm{H}-\mathrm{NMR}$ data for the compounds $(46,48,51,54,55,57,61,63)$ are shown in Table (5) and the ${ }^{13} \mathrm{C}$-NMR data for the compounds $(57,60,64,66,69,70,72,73)$ are shown in Table (6) . The (UV) spectra showed ( $\lambda$ max) in the range of (290-354) $\mathrm{nm}$.

\section{REFERENCES}

Al- Hajjar, F.H.; Jarrar, A.A. (1980). Reactions of $\alpha$, $\beta$-unsaturated ketones with cynoacetamide. $J$. Heterocyclic Chem., 17, 1521,

El-Gazzar, A.R.B.A.; Hussein, H.A.R.; Hafez, H.N. (2007). Synthesis and biological evaluation of thieno[2,3-d]pyrimidine derivatives for anti-inflammatory, analgesic and ulcerogenic activity. Acta Pharm. 57, 395-411.

Abolwafa, O.M.; Ismail, K.A.; Koreish, E.A. (1992). Synthesis and antimicrobial activity of some thiazolinyl teteahydrobenzo[b]thiophenes and thiazolinyl tetrahydrobenzothiene [2,3d]pyrimidine-4-ones. Farmaco., 47, 631-642.

Attab, F.A.; Eldin, S.M. (1999). Synthesis of pyrimidine, thiazolopyrimidine, pyrimidinotriazine and triazolopyrimidine derivatives and their biological evaluations. Natur Forsch, 54b , 788.

Misha, R.; Mishra, B.M.; Moorth, H.A. (2008). Synthesis and antimicrobial evaluation of some 3,4dihydropyrimidine-2-one derivatives. Trends in Appl. Sci. Research. 3(2), 203-208

Kappe, C.O. (1993). 100 Years of the biginelli dihydropyrimidine synthesis Tetrahedron, 49(32), 6937-6963,

Kappe, C.O. (1997). Dipolar cycloaddition reaction of dihydropyrimidine-fused mesomeric betains.An approach toward conformationally restricted dihydropyrimidine derivatives. $J$. Org. Chem., 62, 3109-3118.

Krein, E.B.; Aizenshtat, Z. (1993). Phase-transpher-catalyzed reactions between polysulfide amines and $\alpha, \beta$-unsaturated carbonyl compounds. J. Org. Chem., 58, 6103-6108,

Marvel, C.S.; Coleman, L.E.; Scott, G.P. (1992). Pyrimidine analogs of chalcone and their polymerization reactions. J. Org. Chem., 57, 1785-1792,

Howells, R.E.; Tinsley, E.; Devaney, E.; Smith, G. (1981). The effect of 5-fluorouracil and 5fluorocyctosine on the development of the filarial nematodes. Acta, Trop., 38, 289-304

Vogel, A.I. (1981). "Text Book of Practical Chemistry". 3rd edn. Longman group, London, 79 p. 